\title{
Biomechanical Analysis of Stand-alone Lateral Lumbar Interbody Fusion for Lumbar Adjacent Segment Disease
}

Michael Chioffe ${ }^{1}$, Michael McCarthy ${ }^{2}$, Peter R. Swiatek ${ }^{3}$, Joseph P. Maslak ${ }^{4,} 3$, Leonard I. Voronov ${ }^{5}$, Robert M. Havey ${ }^{3}$, Muturi Muriuki ${ }^{6}$, Avinash Patwardhan ${ }^{7}$, Alpesh A. Patel ${ }^{3}$

1. Orthopaedic Surgery, Sarah Bush Lincoln Health Center, Mattoon, USA 2. Orthopaedics, Spine Surgery, Hospital for Special Surgery, New York, USA 3. Orthopaedic Surgery, Northwestern Memorial Hospital, Chicago, USA 4. Orthopaedics, Spine Surgery, Cleveland Clinic, Cleveland, USA 5. Orthopaedic Surgery, Loyola University Chicago, Maywood, USA 6. Orthopaedic Surgery, Edward Hines, Jr. Veterans Administration Hospital, Hines, USA 7. Orthopaedic Surgery, Loyola University Medical Center, Chicago, USA

Corresponding author: Michael McCarthy, michaelhallmccarthy@gmail.com

\section{Abstract}

\section{Study design}

Biomechanical cadaveric study

\section{Objective}

To compare biomechanical properties of a single stand-alone interbody fusion and a single-level pedicle screw construct above a previous lumbar pedicle fusion.

\section{Summary of background data}

Adjacent segment disease (ASD) is spondylosis of adjacent vertebral segments after previous spinal fusion. Despite the consensus that ASD is clinically significant, the surgical treatment of ASD is controversial.

\section{Methods}

Lateral lumbar interbody fusion (LLIF) and posterior spinal fusion (PSF) with pedicle screws were analyzed within a validated cadaveric lumbar fusion model. L3-4 vertebral segment motion was analyzed within the following simulations: without implants (intact), L3-4 LLIF-only, L3-4 LLIF with previous L4-S1 PSF, L3-4 PSF with previous L4-S1 PSF, and L4-S1 PSF alone. L3-4 motion values were measured during flexion/extension with and without axial load, side bending, and axial rotation.

\section{Results}

Received 09/23/2019 Review began 11/01/2019 Review ended 11/15/2019 Published 11/20/2019

๑) Copyright 2019 Chioffe et al. This is an open access article distributed under the terms of the Creative Commons Attribution License CC-BY 3.0., which permits unrestricted use, distribution, and reproduction in any medium, provided the original author and source are credited.
L3-4 motion in the intact model was found to be $4.7 \pm 1.2$ degrees. L3-4 LLIF-only decreased motion to $1.9 \pm$ 1.1 degrees. L3-4 LLIF with previous L4-S1 fusion demonstrated less motion in all planes with and without loading ( $\mathrm{p}<0.05$ ) compared to an intact spine. However, L3-4 motion with flexion/extension and lateral bending was noted to be greater compared to the L3-S1 construct $(\mathrm{p}<0.5)$. The L3-S1 PSF construct decreased motion to less than $1^{\circ}$ in all planes of motion with or without loading $(\mathrm{p}<0.05)$. The L3-4 PSF with previous L4-S1 PSF constructs decreased the flexion/extension motion by $92.4 \%$ compared to the intact spine, whereas the L3-4 LLIF with previous L4-S1 PSF constructs decreased motion by $61.2 \%$.

\section{Conclusions}

Stand-alone LLIF above a previous posterolateral fusion significantly decreases motion at the adjacent segment, demonstrating its utility in treating ASD without necessitating revision. The stand-alone LLIF is a biomechanically sound option in the treatment of ASD and is advantageous in patient populations who may benefit from less invasive surgical options.

Categories: Neurosurgery, Orthopedics

Keywords: interbody fusion, posterior spinal fusion, adjacent segment disease, spondylosis, biomechanics

\section{Introduction}

Adjacent segment disease (ASD) is a postoperative diagnosis in patients with prior histories of spinal fusion. A diagnosis of ASD is predicated on both adjacent segment radiographic degeneration and the development of clinical symptoms. Multiple longitudinal studies have demonstrated that radiographic evidence of adjacent segment degeneration is not directly correlated with ASD, as some radiographic findings can be nonspecific and not clinically relevant [1-3]. Numerous studies have identified multiple risk factors for the 


\section{Cureus}

development of ASD, including age over 60 years, male gender, facet degeneration, multilevel fusion, fusion to L5, and preexisting degenerative disc disease adjacent to the fused segment [4-8].

Treatment of symptomatic ASD traditionally includes surgical decompression and extension of the existing posterior instrumentation and fusion. The approach to revising posterior spinal fusions is often more complex than the index procedure and has been associated with increased complications and poor outcomes $[2,9-10]$. The difficulty of revision posterior spinal fusion often leads to the use of minimally invasive interbody fusion. Anterior, oblique, and lateral retroperitoneal approaches have become increasingly popular for the treatment of symptomatic ASD [11-12]. The direct lateral approach is an effective means for both successful arthrodesis and indirect decompression [11, 13-14].

While the use of lateral lumbar interbody fusion (LLIF) for ASD has been reported in recent literature, LLIF alone has not been rigorously investigated in a cadaveric model [15-16]. The adjacent segment is a difficult biomechanical environment for fusion with increased stresses and abnormal motion patterns due to the lack of mobility. The purpose of this study was to compare biomechanical properties of a single stand-alone interbody fusion and a single-level pedicle screw construct above a previous lumbar pedicle fusion.

\section{Materials And Methods}

Six fresh-frozen human lumbar spine specimens (T10-S1) were used for this study with the demographics of each listed in Table 1.

\begin{tabular}{|c|c|c|c|}
\hline Specimen & Age (years) & Sex & Cause of Death \\
\hline 1 & 52 & M & Respiratory Distress Syndrome \\
\hline 2 & 39 & M & Lung Cancer \\
\hline 3 & 44 & M & Cirrhosis of the Liver \\
\hline 4 & 49 & $\mathrm{~F}$ & Drug Overdose \\
\hline 5 & 51 & $\mathbf{F}$ & Diabetic Shock \\
\hline 6 & 32 & M & GSW to the Head \\
\hline Mean & 44.5 & & \\
\hline SD & 7.8 & & \\
\hline
\end{tabular}

\section{TABLE 1: Specimen Demographics}

GSW: gunshot wound; SD: standard deviation

Radiographic screening was performed to exclude specimens with fractures, metastatic disease, bridging osteophytes, osteoporosis, previous spine surgeries, or other conditions that could significantly affect the biomechanics of the spine. The specimen was thawed and stripped of the paraspinal musculature while preserving the discs, facet joints, and osteoligamentous structures.

All biomechanical testing was performed at room temperature. The specimen was fixed to the apparatus at the caudal end and free to move in any plane at the proximal end. The apparatus allowed continuous cycling of the specimen between specified maximum moment endpoints in flexion-extension, lateral bending, and axial rotation (Figure 1). 


\section{Cureus}
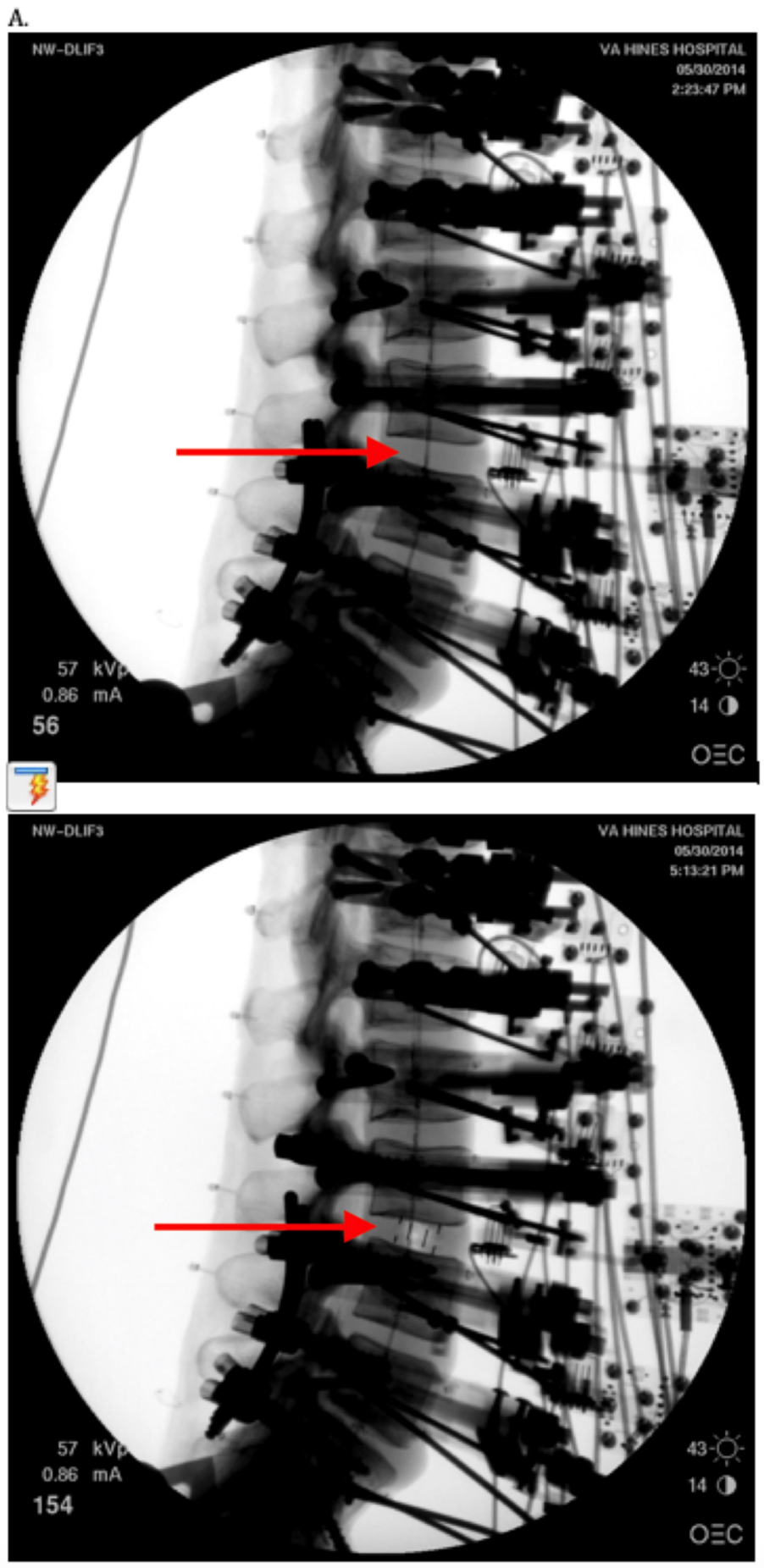

FIGURE 1: Radiographic images before and after interbody spacer placement

A) X-ray images of the cadaver spine specimen before interbody spacer placement; B) X-ray images of the cadaver spine specimen after interbody spacer placement

The load-displacement data was collected until two reproducible load-displacement loops were obtained.

The angular motion of the T10 to S1 vertebrae was measured using an optoelectronic motion measurement system (Optotrak Certus ${ }^{\circledR}$, Northern Digital, Inc., Waterloo, Ontario, Canada) (Figure 2). 


\section{Cureus}

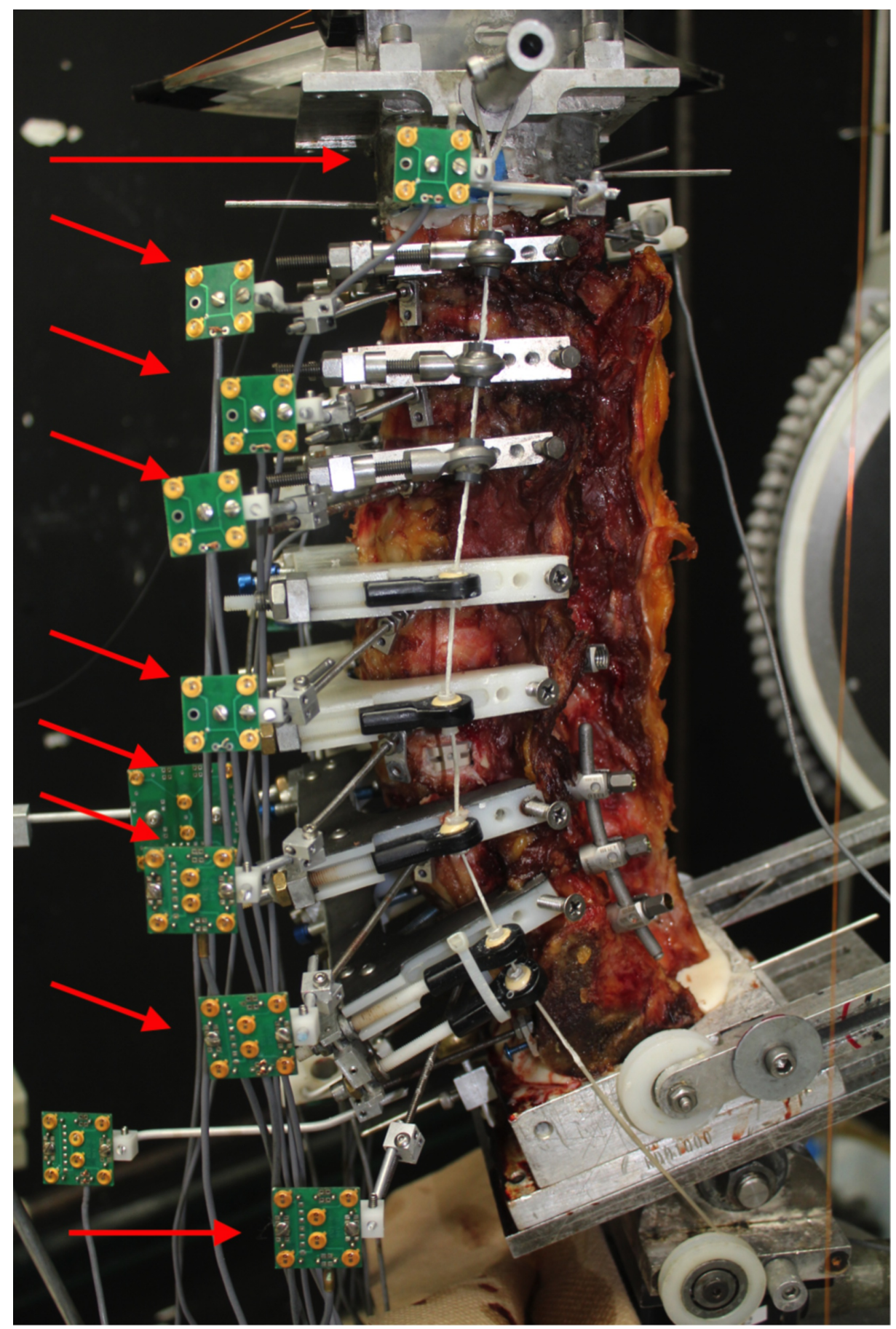

FIGURE 2: Photograph of the lumbar spine specimen with motion analysis sensors in place

In addition, biaxial angle sensors (Model 902-45 Biaxial Clinometer) (Applied Geomechanics, Inc., Santa Cruz, CA) were mounted on each vertebra to allow real-time feedback for the optimization of the preload path. A six-component load cell (Model MC3A-6-1000) (AMTI, Inc., Watertown, MA) was placed under the specimen to measure the applied compressive preload and moments. Fluoroscopic imaging (General Electric OEC ${ }^{\circledR} 9800$ Plus digital fluoroscopy machine) (GE Healthcare, Chicago, IL) was used during implantation to ensure proper implant placement. Fluoroscopic images of the mobile segments were taken in the neutral, flexed, and extended postures during the kinematic testing.

Each specimen's intact range of motion (ROM) was tested in flexion-extension, lateral bending, and axial rotation under moment control using $8 \mathrm{~N}$ (newtons) flexion and $6 \mathrm{~N}$ extension moments with no preload and then with $400 \mathrm{~N}$ following preload. Each specimen was tested using the following stepwise protocol: intact, L4-S1 posterior spinal fusion (PSF), L3-S1 PSF, L4-S1 PSF with LLIF at L3-4, then LLIF at L3-4 without PSF. 
Following intact testing, a fusion was performed at L4-S1 using posterior pedicle screws with rods. Bilateral pedicle screws and rods were then extended to the L3-L4 segment and the previous load control protocol was repeated. Next, the lateral interbody cage was implanted at the L3-L4 segment and the previous load control protocol was repeated. The lateral technique involved the preservation of the anterior longitudinal ligament but with the release of the ipsilateral and contralateral annulus. The final step of the protocol was testing a stand-alone LLIF at L3-L4 after removing posterolateral fusion.

\section{Results}

Following posterolateral fusion at L4-S1, the ROM at L3-L4 increased compared to the intact condition in flexion-extension, lateral bending, and axial rotation but did not reach statistical significance $(\mathrm{p}>0.05)$ (Table 2).

\begin{tabular}{|c|c|c|c|c|c|}
\hline L3-L4 ROM (degrees) & Intact & L4-S1 Fusion & L3-S1 Fusion & L4-S1 Fusion, DLIF at L3-L4 & Stand-alone DLIF at L3-L4 \\
\hline Flex-Ext ON & $4.7 \pm 1.2$ & $5.4 \pm 1.3$ & $0.5 \pm 0.2^{*}$ & $2.0 \pm 0.9^{*} \dagger$ & $1.9 \pm 1.1$ \\
\hline Flex-Ext 400N & $5.2 \pm 1.0$ & $5.7 \pm 1.1$ & $0.4 \pm 0.1^{*}$ & $2.1 \pm 0.8^{*} \dagger$ & $1.8 \pm 1.0$ \\
\hline Lateral Bending & $8.2 \pm 1.7$ & $8.3 \pm 1.7$ & $0.6 \pm 0.1^{*}$ & $4.0 \pm 1.8^{*} \dagger$ & $4.6 \pm 2.0$ \\
\hline Axial Rotation & $1.4 \pm 0.7$ & $1.3 \pm 0.8$ & $0.5 \pm 0.2^{\star}$ & $0.7 \pm 0.3^{*}$ & $1.3 \pm 0.8$ \\
\hline
\end{tabular}

TABLE 2: The L3-L4 Range of Motion Values (Mean \pm SD) During Flexion-Extension (0N and 400N), Lateral Bending, and Axial Rotation

( ${ }^{*}$ denotes significant difference from intact, $\mathrm{p}<0.05$ )

(† denotes significant difference from L3-S1 fusion, $\mathrm{p}<0.05$ )

DLIF: direct lateral interbody fusion; Ext: extension; Flex: flexion; N: newtons; ROM: range of motion; SD: standard deviation

When the posterolateral fusion was extended to L3, there was a significant decrease in L3-L4 motion (less than one degree) in all-loading modes $(\mathrm{p}<0.05)$. When posterolateral fusion at L3-L4 was replaced by a stand-alone LLIF (above a fusion at L4-S1), the ROM at L3-L4 continued to be significantly lower than intact ( $p<0.05$ in all loading modes) but was also significantly greater than that of a posterolateral fusion at L3-L4 ( $\mathrm{p}<0.05$ for flexion-extension and lateral bending, $\mathrm{p}=0.11$ for axial rotation). Overall, the L3-S1 pedicle screw fusion constructs decreased the flexion-extension ROM at $400 \mathrm{~N}$ by $92.0 \%$, whereas the L3- 4 LLIF and L4-S1 fusion construct decreased flexion-extension ROM by 58.6\% of intact. Removal of a posterolateral fusion at L4-S1, leaving a stand-alone direct lateral interbody fusion (DLIF) at L3-L4, did not significantly change the motion at L3-L4 ( $p>0.05)$.

\section{Discussion}

The adjacent level of a prior posterior spinal fusion is a biomechanically and biologically challenging environment [17]. The lateral approach offers a minimally invasive method of fusion while avoiding the complications associated with revision posterior spinal fusion [18]. In addition to avoiding these complications, the LLIF can address underlying foraminal and central stenosis via indirect decompression $[8$, 19]. Recent studies have demonstrated the capabilities of lateral interbody fusion in the treatment of ASD [6, 20-21]. LLIF is an attractive alternative in treating ASD due to limited blood loss, no need to reexplore previous laminectomies, and an overall reduction in complications [6]. Although LLIF has been demonstrated to be a viable alternative to PSF, there still lacks a significant body of literature supporting its use as the gold standard in treating ASD [22].

This biomechanical study demonstrates a statistically significant reduction in motion in all load parameters and all axes of motion with a stand-alone lateral interbody fusion adjacent to a prior posterolateral fusion. Although our model demonstrated less rigidity (56\% loss of motion) with the interbody construct compared to the posterior instrumented fusion ( $92 \%$ loss of motion), it remains an attractive option in the treatment of ASD.

Within our study, the results of extending the PSF in the treatment of ASD provide evidence of this construct's ability to provide increased structural rigidity. Our outcomes demonstrate a significant decrease in L3-L4 motion in all-loading modes ( $\mathrm{p}<0.05)$; however, this study did not assess the significance of this increased stability in comparison to the LLIF construct. Results demonstrated that the LLIF construct provides a significant reduction in motion compared to an intact spine; however, this study was unable to assess superiority between revision PSF and LLIF. The tenants of orthopedic surgery and spine fusion 
surgery are predicated on reducing motion, thus increasing a construct's ability to aid in fusion; therefore, increased rigidity can be correlated with a likely increased fusion mass. Assessing the association between rigidity and likelihood of fusion is outside the scope of this study; thus, one limitation of our study is its inability to quantify significant motion reduction to a fusion between the two constructs.

This study confirms the stability provided by a stand-alone LLIF construct for the treatment of adjacent segment disease above a previous lumbar fusion. In addition to avoiding the complication associated with posterior revision surgery, the LLIF's rigidity and large intervertebral fusion bed make it an attractive option in the treatment of ASD. Recent studies have demonstrated LLIF's viability as a treatment modality for ASD; however, large scale prospective studies are needed to further delineate potential benefits and indications for use of this stand-alone interbody construct for this disease [15, 20].

\section{Conclusions}

Recent advancements with the implementation of interbody fusion constructs and their versatility within spinal surgery have made for an attractive option in minimally invasive spine fusion. In the setting of ASD, interbody constructs, specifically, LLIF, are powerful tools for surgeons in need of fusion options while avoiding the complications associated with posterior revision surgery. Our study provides in vitro biomechanical evidence demonstrating a significant reduction of motion at the level adjacent to a prior posterior spinal fusion. The LLIF is a biomechanically sound option for fusion in the treatment of ASD and is advantageous in patient populations who would benefit from minimally invasive surgery.

\section{Additional Information \\ Disclosures}

Human subjects: Consent was obtained by all participants in this study. Animal subjects: All authors have confirmed that this study did not involve animal subjects or tissue. Conflicts of interest: In compliance with the ICMJE uniform disclosure form, all authors declare the following: Payment/services info: All authors have declared that no financial support was received from any organization for the submitted work. Financial relationships: All authors have declared that they have no financial relationships at present or within the previous three years with any organizations that might have an interest in the submitted work. Other relationships: All authors have declared that there are no other relationships or activities that could appear to have influenced the submitted work.

\section{References}

1. Kumar MN, Jacquot F, Hall H: Long-term follow-up of functional outcomes and radiographic changes at adjacent levels following lumbar spine fusion for degenerative disc disease. Eur Spine J. 2001, 10:309-13. $10.1007 /$ s005860000207

2. Hilibrand AS, Robbins M: Adjacent segment degeneration and adjacent segment disease: the consequences of spinal fusion?. Spine J. 2004, 4:S190-94. 10.1016/j.spinee.2004.07.007

3. Ghiselli G, Wang JC, Bhatia NN, Hsu WK, Dawson EG: Adjacent segment degeneration in the lumbar spine . J Bone Joint Surg Am. 2004, 86:1497-503. 10.2106/00004623-200407000-00020

4. Axelsson P, Johnsson R, Strömqvist B: Adjacent segment hypermobility after lumbar spine fusion: no association with progressive degeneration of the segment 5 years after surgery. Acta Orthop. 2007, 78:83439. 10.1080/17453670710014635

5. Etebar S, Cahill DW: Risk factors for adjacent-segment failure following lumbar fixation with rigid instrumentation for degenerative instability. J Neurosurg. 1999, 90:163-69. 10.3171/spi.1999.90.2.0163

6. Wang MY, Vasudevan R, Mindea SA: Minimally invasive lateral interbody fusion for the treatment of rostral adjacent-segment lumbar degenerative stenosis without supplemental pedicle screw fixation. J Neurosurg Spine. 2014, 21:861-66. 10.3171/2014.8.SPINE13841

7. Ahmadian A, Verma S, Mundis GM Jr, Oskouian RJ Jr, Smith DA, Uribe JS: Minimally invasive lateral retroperitoneal transpsoas interbody fusion for L4-5 spondylolisthesis: clinical outcomes. J Neurosurg Spine. 2013, 19:314-20. 10.3171/2013.6.SPINE1340

8. Alimi M, Hofstetter CP, Cong GT, et al.: Radiological and clinical outcomes following extreme lateral interbody fusion. J Neurosurg Spine. 2014, 20:623-35. 10.3171/2014.1.SPINE13569

9. Cheh G, Bridwell KH, Lenke LG, Buchowski JM, Daubs MD, Kim Y, Baldus C: Adjacent segment disease followinglumbar/thoracolumbar fusion with pedicle screw instrumentation: a minimum 5-year follow-up. Spine (Phila Pa 1976). 2007, 32:2253-57. 10.1097/BRS.0b013e31814b2d8e

10. Castellvi AE, Nienke TW, Marulanda GA, Murtagh RD, Santoni BG: Indirect decompression of lumbar stenosis with transpsoas interbody cages and percutaneous posterior instrumentation. Clin Orthop Relat Res. 2014, 472:1784-91. 10.1007/s11999-014-3464-6

11. Abbasi H, Abbasi A: Minimally invasive direct lateral interbody fusion (MIS-DLIF): proof of concept and perioperative results. Cureus. 2017, 9:e979. 10.7759/cureus.979

12. Fogel GR, Parikh RD, Ryu SI, Turner AW: Biomechanics of lateral lumbar interbody fusion constructs with lateral and posterior plate fixation: laboratory investigation. J Neurosurg Spine. 2014, 20:291-97. 10.3171/2013.11.SPINE13617

13. Campbell PG, Nunley PD, Cavanaugh D, Kerr E, Utter PA, Frank K, Stone M: Short-term outcomes of lateral lumbar interbody fusion without decompression for the treatment of symptomatic degenerative spondylolisthesis at L4-5. Neurosurg Focus. 2018, 44:E6. 10.3171/2017.10.FOCUS17566

14. Jin J, Ryu KS, Hur JW, Seong JH, Kim JS, Cho HJ: Comparative study of the difference of perioperative 


\section{Cureus}

complication and radiologic results: MIS-DLIF (minimally invasive direct lateral lumbar interbody fusion) versus MIS-OLIF (minimally invasive oblique lateral lumbar interbody fusion). Clin Spine Surg. 2018, 31:3136. 10.1097/BSD.0000000000000474

15. Metzger MF, Robinson ST, Maldonado RC, Rawlinson J, Liu J, Acosta FL: Biomechanical analysis of lateral interbody fusion strategies for adjacent segment degeneration in the lumbar spine. Spine J. 2017, 17:100411. 10.1016/j.spinee.2017.03.005

16. Louie PK, Varthi AG, Narain AS, Lei V, Bohl DD, Shifflett GD, Phillips FM: Stand-alone lateral lumbar interbody fusion for the treatment of symptomatic adjacent segment degeneration following previous lumbar fusion. Spine J. 2018, 18:2025-32. 10.1016/j.spinee.2018.04.008

17. Gillet P: The fate of the adjacent motion segments after lumbar fusion . J Spinal Disord Tech. 2003, 16:33845. 10.1097/00024720-200308000-00005

18. Pawar A, Hughes A, Girardi F, Sama A, Lebl D, Cammisa F: Lateral lumbar interbody fusion. Asian Spine J. 2015, 9:978-83. 10.4184/asj.2015.9.6.978

19. Yoshihara H: Indirect decompression in spinal surgery. J Clin Neurosci. 2017, 44:63-68. 10.1016/j.jocn.2017.06.061

20. Aichmair A, Alimi M, Hughes AP, et al.: Single-level lateral lumbar interbody fusion for the treatment of adjacent segment disease: a retrospective two-center study. Spine (Phila Pa 1976). 2017, 42:e515-22. 10.1097/BRS.0000000000001871

21. Louie PK, Haws BE, Kahn JM, et al.: Comparison of stand-alone lateral lumbar interbody fusion versus open laminectomy and posterolateral instrumented fusion in the treatment of adjacent segment disease following previous lumbar fusion surgery. Spine (Phila Pa 1976). 2019, Aug:Epub ahead of print. 10.1097/BRS.0000000000003191

22. Palejwala SK, Sheen WA, Walter CM, Dunn JH, Baaj AA: Minimally invasive lateral transpsoas interbody fusion using a stand-alone construct for the treatment of adjacent segment disease of the lumbar spine: review of the literature and report of three cases. Clin Neurol Neurosurg. 2014, 124:90-96. 10.1016/j.clineuro.2014.06.031 